\title{
RETROGRADE LEFT VENTRICULAR CATHETERIZATION AND CARDIOANGIOGRAPHY IN AORTIC STENOSIS
}

\author{
BY \\ I. R. GRAY AND C. S. JOSHIPURA \\ From Birmingham Regional Thoracic Centre, Hertford Hill, Nr. Warwick \\ Received May 28, 1963
}

It is often difficult to judge the severity of aortic stenosis by clinical methods and to distinguish between the different varieties that occur. The size of the orifice can be calculated by measuring aortic and left ventricular pressures and simultaneously estimating the cardiac output. The site of the obstruction may be demonstrated by recording the changes in pressure as a catheter passes from the left ventricle to the aorta. Cardioangiography after injection of contrast medium into the left atrium or ventricle can also demonstrate visibly the site and nature of the obstruction.

Retrograde catheterization of the left ventricle from a peripheral artery, with injection of contrast medium into the left ventricle, is a simple means of obtaining all the required information. We describe our results in a series of patients with aortic stenosis investigated in this way.

\section{SubJeCtS AND Methods}

Twenty-five patients with signs of significant aortic stenosis were investigated. The aortic lesion was congenital in 14 and rheumatic in 7, all of whom had associated lesions of the mitral valve. One patient had hypertrophic muscular sub-aortic stenosis, and the ætiology in three patients with aortic valve stenosis was uncertain (Table).

Right cardiac catheterization was first carried out and the catheter left with its tip in the pulmonary artery. The right brachial artery or one of its branches was then catheterized using a thin-walled nylon catheter (N.I.H. pattern Nos. 6-9). The passage of the catheter was followed radiologically using an image intensifier, and the pressure was monitored on an oscilloscope which immediately showed when the ventricle was entered. The catheter was changed for one of different shape and size if there was difficulty in passing it through the orifice of the aortic valve. As soon as the left ventricle was catheterized the oxygen consumption was measured and the cardiac output estimated by the Fick principle.

TABLE

Diagnosis in 25 Patients with Aortic Stenosis

\begin{tabular}{|c|c|c|c|c|c|c|c|}
\hline & & & & & Congenital & Rheumatic & Unknown \\
\hline $\begin{array}{l}\text { Valve .. } \\
\text { Subvalvar diaphragm.. } \\
\text { Supravalvar narrowing } \\
\text { Combined valvar and s } \\
\text { Combined valvar and s } \\
\text { Muscular subvalvar .. }\end{array}$ & $\begin{array}{l}\cdots \\
\cdots \\
\text { avalvar } \\
\text { alvar } \\
\ldots\end{array}$ & $\begin{array}{l}\cdots \\
\cdots \\
\cdots \\
\cdots \\
\cdots\end{array}$ & $\begin{array}{l}\cdots \\
\cdots \\
\cdots \\
\cdots \\
\cdots\end{array}$ & $\begin{array}{l}\cdots \\
\cdots \\
\cdots \\
\cdots \\
\cdots\end{array}$ & $\begin{array}{r}10 \\
1 \\
1 \\
1 \\
1 \\
-\end{array}$ & $\begin{array}{l}7 \\
= \\
= \\
-\end{array}$ & $\frac{3}{-}$ \\
\hline Total. . & . & . & . & $\ldots$ & 14 & 7 & 4 \\
\hline
\end{tabular}


Cardioangiography was then carried out, with or without light anæsthesia, injecting Hypaque 0.5-1 ml. per kg. body weight. The 85 per cent solution was used in adults and 65 per cent in children. Films were exposed either in a single plane using a right posterior oblique position or with simultaneous antero-posterior and lateral exposures. Four or five films were exposed each second for six seconds. The catheter was then slowly withdrawn to the aorta, the pressure being recorded continuously.

The radiograms were studied to judge the appearance and mobility of the aortic valve and to detect any obstruction in the ascending aorta or in the left ventricle. The size of the left ventricle in systole and diastole was estimated, and the appearance of the coronary arteries and the ascending aorta examined.

If the catheter could not be introduced into the left ventricle, contrast was injected into the ascending aorta and serial radiograms taken, and the left ventricular pressure subsequently measured by left ventricular puncture.

\section{RESULTS}

The left ventricle was catheterized in 21 of the 25 patients: the 4 failures were in patients with severe aortic valve stenosis. In cases other than aortic stenosis the left ventricle has been successfully catheterized in 41 of 44 attempts. Ischæmic change in the electrocardiogram, serious ventricular arrhythmia, and penetration of the myocardium by injected contrast medium were complications that were encountered early in our experience of left ventricular cardioangiography (Gray, Joshipura, and Mackinnon, 1963), but in the present series of investigations none of these occurred.

Peak systolic pressure gradients in our patients ranged from 5 to $130 \mathrm{~mm}$. $\mathrm{Hg}$ (mean 53). The site of obstruction could often be recognized as the catheter entered the ventricle but was more clearly shown during slow withdrawal. The stenosis was thought to be at the level of the valve in 20 patients including the 4 in whom ventricular catheterization failed. In valvar stenosis the change in systolic pressure coincided with the change from left ventricular to aortic diastolic pressure (Fig. 1).

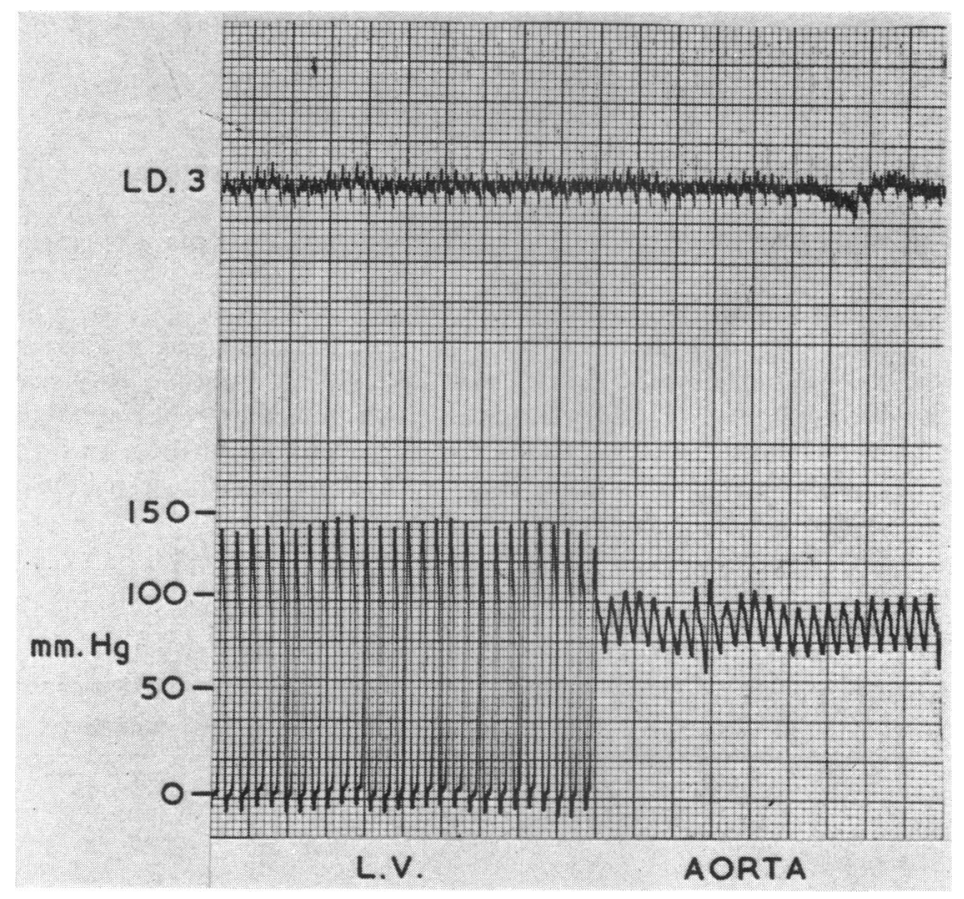

FIG. 1.-Pressure recording from catheter withdrawn from left ventricle to aorta in aortic valvar stenosis. A woman of 25 with congenital aortic stenosis, left ventricular pressure $145 /-5$, and aortic pressure 95/70 mm. Hg. 


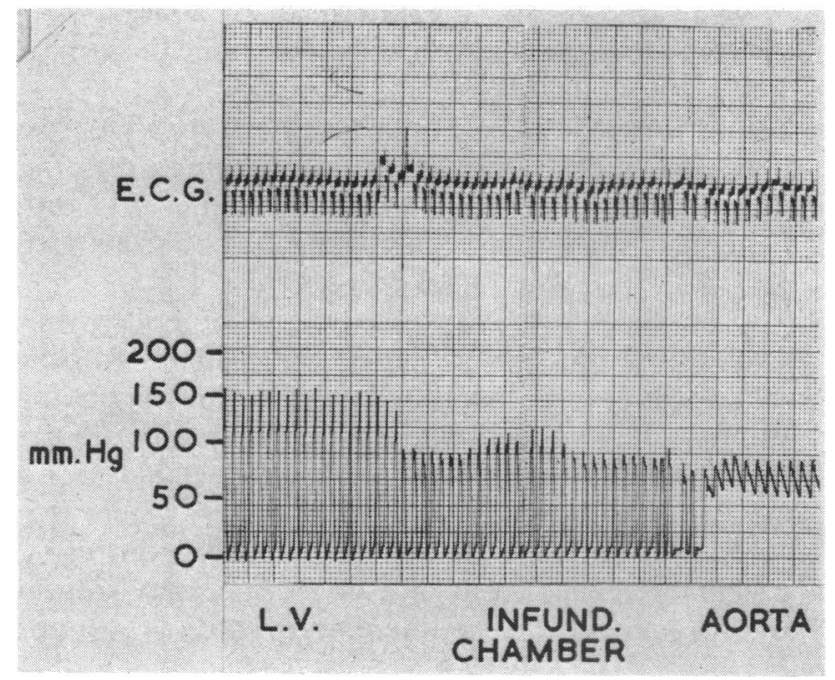

Fig. 2.-Pressure recording from catheter withdrawn from left ventricle to aorta in subvalvar stenosis. Girl of 18 with muscular hypertrophic sub-aortic stenosis. Left ventricular pressure $150 / 0$, subvalvar chamber $95 / 0$, aorta 95/70mm. Hg.

Two patients had sub-aortic stenosis, and in these the pressure gradient was recorded below the valve, and there was an intermediate zone with its systolic pressure identical with the aorta and its diastolic pressure identical with that of the ventricle (Fig. 2). The records from the patient with stenosis from muscular hypertrophy were indistinguishable from those recorded in diaphragmatic obstruction.

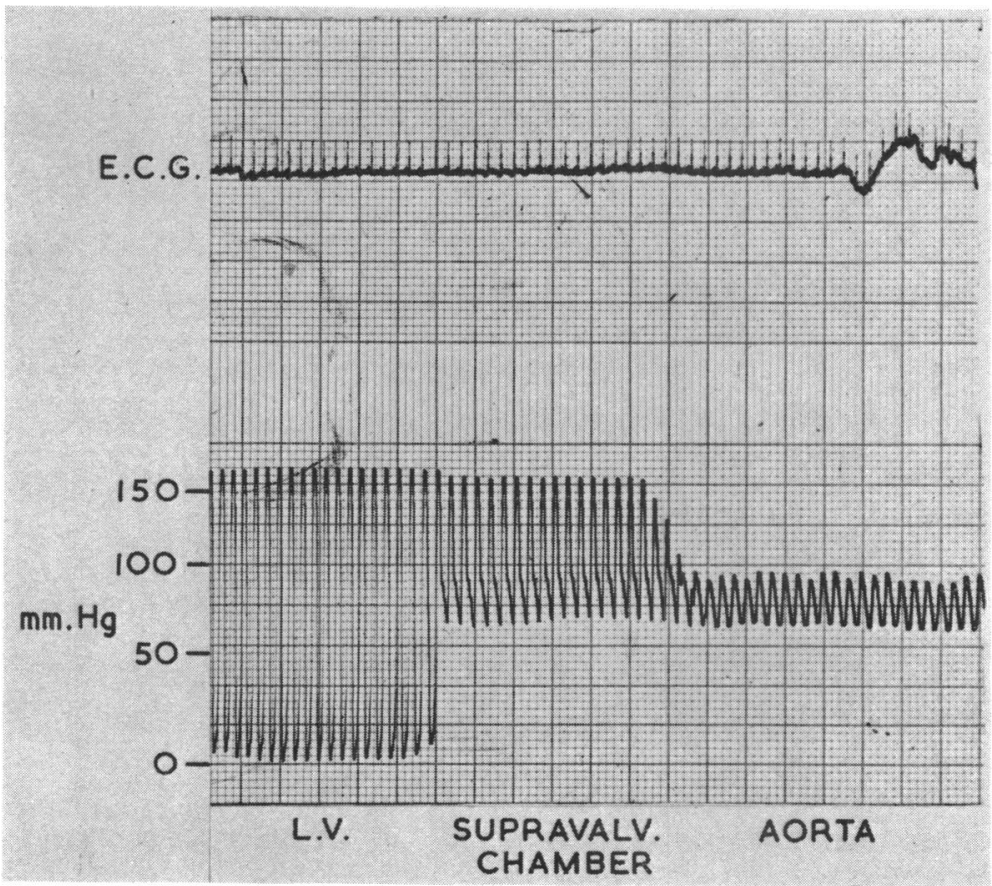

FIG. 3.-Pressure recording from catheter withdrawn from left ventricle to aorta in supravalvar aortic stenosis. Boy of 9 , left ventricular pressure $165 / 0$, supravalvar chamber $160 / 70$, aorta $90 / 70 \mathrm{~mm}$. $\mathrm{Hg}$. 
In one patient with supravalvar stenosis, the intermediate zone lay above the valve with its systolic pressure identical with that of the ventricle and its diastolic the same as in the aorta above the obstruction (Fig. 3).

Cardioangiography demonstrated clearly the site and nature of the stenosis. The muscular hypertrophic form of subvalvar stenosis (Fig. 4) was quite distinct from the diaphragmatic variety (Fig. 5) which could be quite difficult to identify if a thin diaphragm did not lie exactly in the plane of the $\mathrm{X}$ rays. Supravalvar stenosis was seen as a narrowing of the aorta above the origins of the coronary arteries (Fig. 6).

Cardioangiography gave further information about the valve itself when the stenosis was valvar. A normal aortic valve is not seen during systole in left ventricular cardioangiograms. With aortic stenosis mobility of the valve is likely to be inversely related to the severity of the stenosis. This was borne out by our observations. In 8 patients where the peak systolic gradient exceeded $50 \mathrm{~mm}$. $\mathrm{Hg}$ the mobility of the valve was obviously greatly reduced so that its position altered little during the cardiac cycle (Fig. 7A and B). In 7 patients with valve stenosis where the gradient was less than $50 \mathrm{~mm}$. Hg, part at least of the valve moved upwards into the aorta during systole. This often gave rise to a characteristic appearance reminiscent of a dog's ears, only seen in stenosis that was not severe (Fig. 8A and B). This is due to the ballooning upwards of a mobile valve with a central orifice of moderate size.

It was difficult, particularly in severe valve stenosis, to judge whether there were two or three cusps. Aortography is probably better suited to eliciting this information than ventriculography.

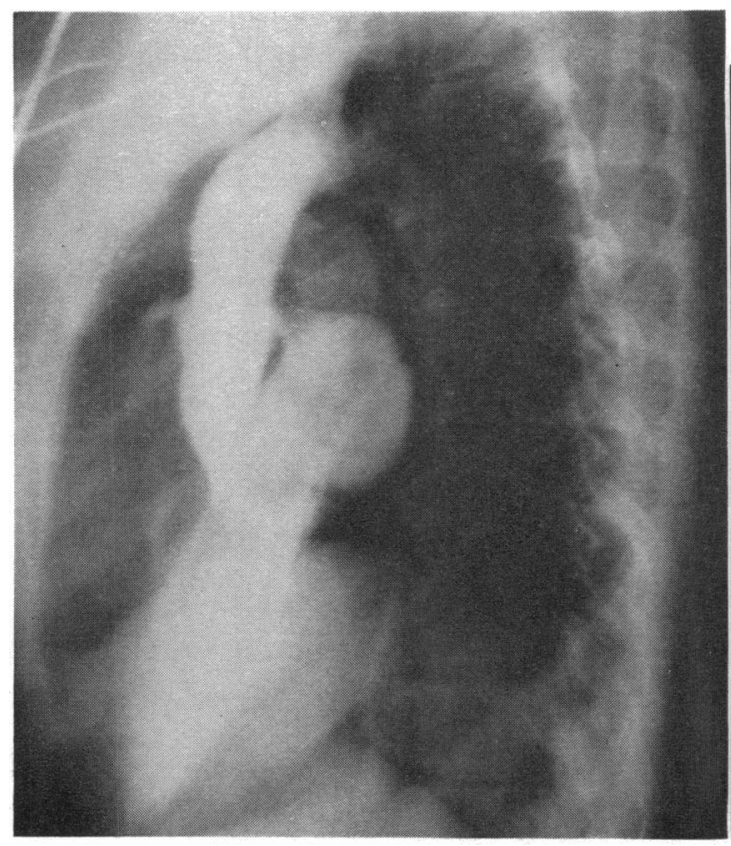

FIG. 4.-Left ventricular cardioangiogram in girl of 18 with muscular hypertrophic sub-aortic stenosis. This film was during systole and shows the constriction of the upper part of the left ventricle as well as opacification of the left atrium from associated mitral regurgitation (same patient as Fig. 2).

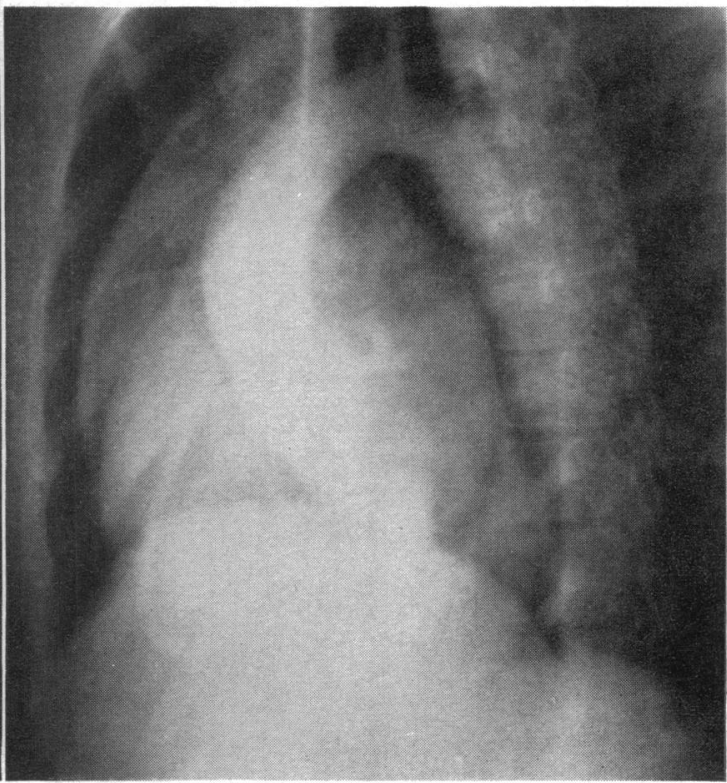

FIG. 5.-Left ventricular cardioangiogram in woman of 29 with diaphragmatic sub-aortic stenosis (left ventricular pressure $205 / 5$, subvalvar chamber $108 / 5$, aorta $110 / 65 \mathrm{~mm}$. Hg). The obstruction is seen as a translucent diaphragm slightly below the aortic valve. 


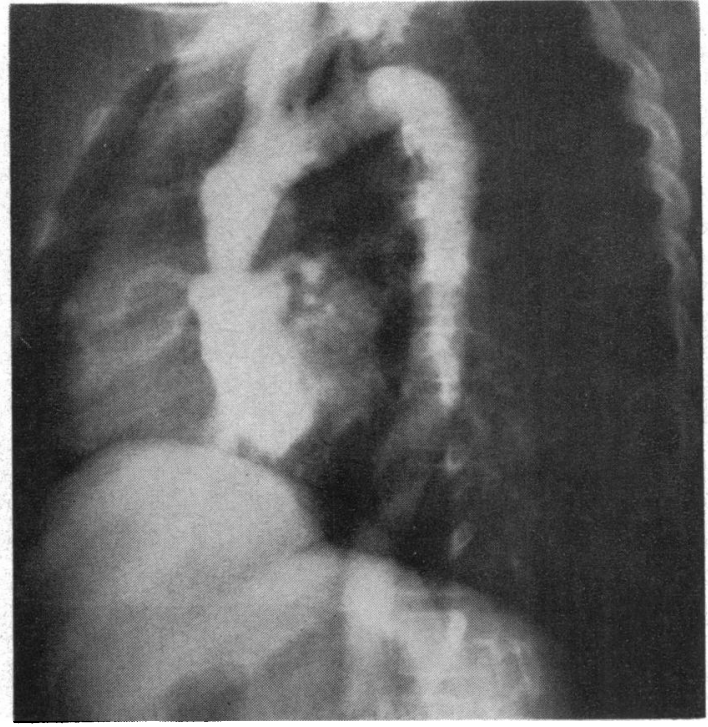

FIG. 6.-Left ventricular cardioangiogram in boy of 9 with supravalvar aortic stenosis (same patient as Fig. 3). A constriction of the left common carotid artery at its origin is also visible.

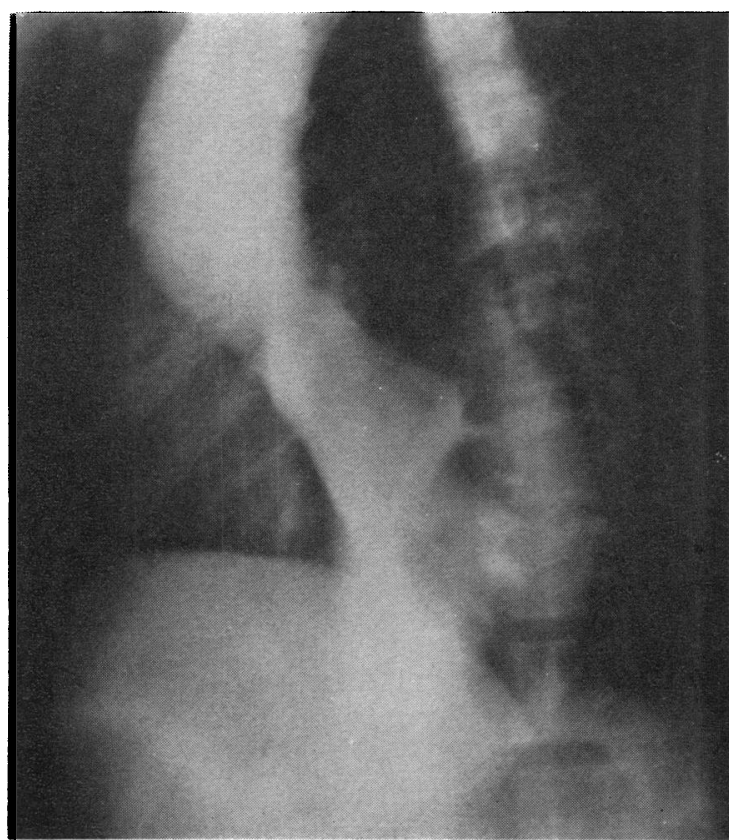

A

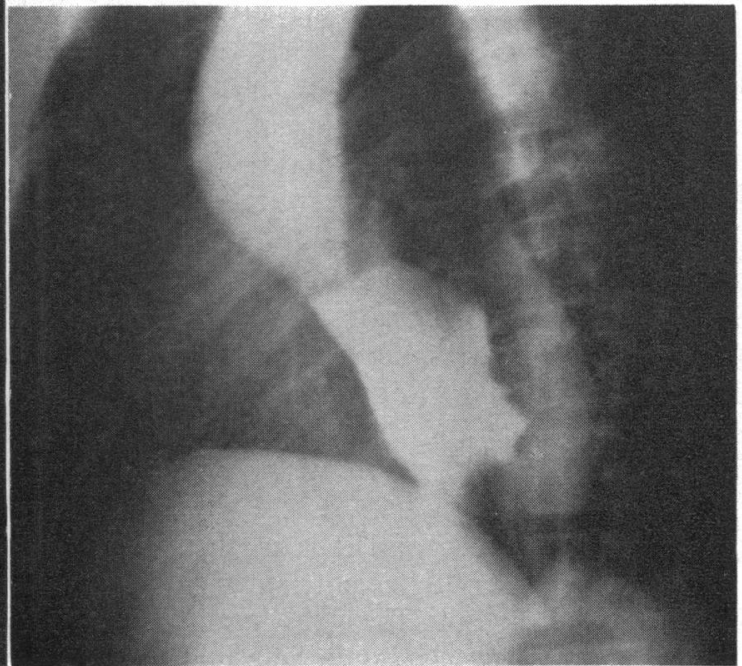

B

FIG. 7A and 7B.-Left ventricular cardioangiograms in systole (A) and diastole (B) in severe aortic valvar stenosis. The greatly reduced mobility of the cusps of the valve is shown together with dilatation of the ascending aorta and absence of coronary arterial filling. 


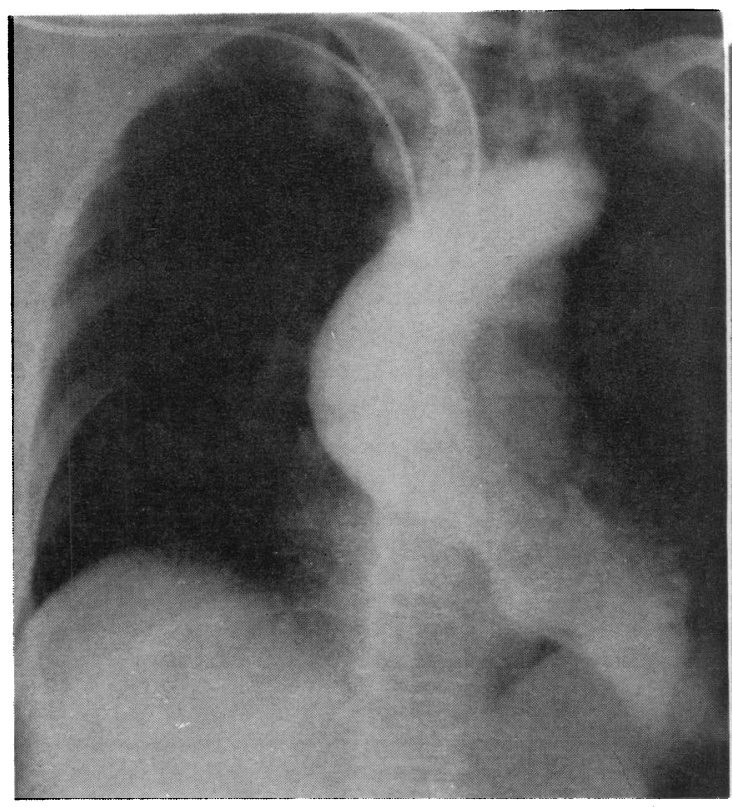

A

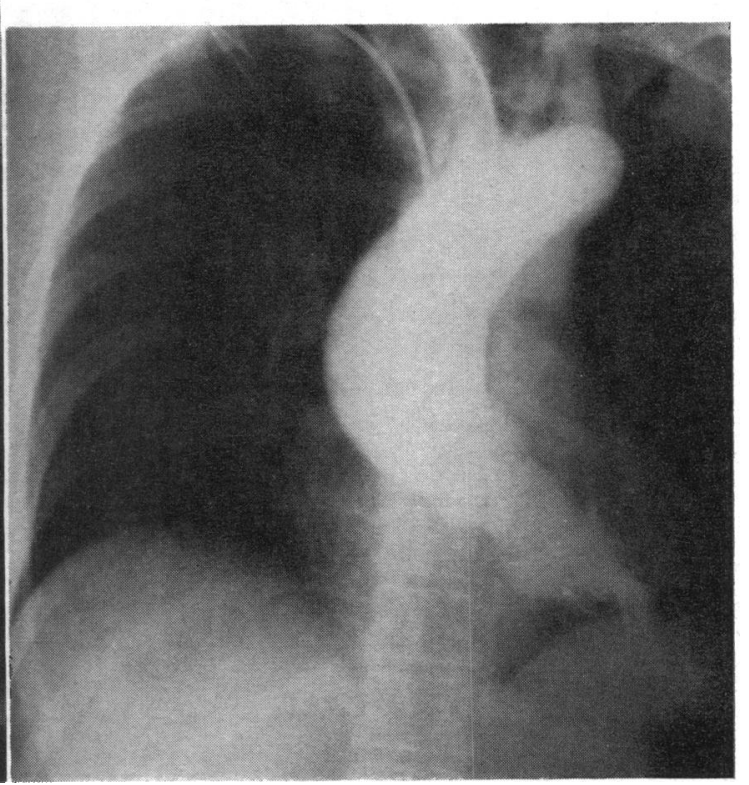

B

FIG. 8A and 8B.--Left ventricular cardiograms in systole (A) and diastole (B) in moderate aortic valvar stenosis. The dog-ear appearance of the aortic cusps is seen in the systolic film and the mobility of the valve is demonstrated by its two positions. There is also an appearance suggesting a subvalvar diaphragm, but this was excluded by the pressure tracings and subsequent surgical exploration.

\section{Discussion}

Catheterization of the left ventricle from a peripheral artery was first described by Zimmerman, Scott, and Becker (1950), and Prioton et al. (1957) reported its use in conjunction with left ventricular cardioangiography in the investigation of mitral regurgitation. The Seldinger technique of arterial catheterization which they employed did not prove satisfactory in patients with aortic stenosis (Stampbach and Joss, 1959; Levy, Amplatz, and Lillehei, 1962). The use of a larger catheter introduced through an incision in the arterial wall has been more successful, and Gründemann et al. (1960) were able to catheterize the left ventricle in 75 per cent of 150 patients with aortic stenosis without any serious complications.

The excellent pressure tracings obtained by this technique demonstrate not only the pressure difference between the aorta and left ventricle but also the site of the change. Together with the left ventricular cardioangiograms these provide most of the information that is needed in making a full assessment of aortic stenosis. In 4 of our 25 patients, however, we were unable to pass a catheter into the ventricle and in several others some difficulty was encountered. The difficult cases, however, were those with severe valvar stenosis in most of whom the presence of calcification in the valve had already been shown. There was seldom any difficulty with congenital stenosis at supravalvar and subvalvar level, and it is in these patients that the information obtained by a withdrawal pressure record and left ventricular cardioangiography is most valuable.

The other methods for investigating aortic stenosis are percutaneous left ventricular puncture and transseptal left ventricular catheterization via the right and left atria. Left ventricular puncture was described by Brock, Milstein, and Ross (1956), and its use was extended by passing a fine catheter through the needle into the aorta (Fleming et al., 1958); this was possible in 78 per cent of cases. A further refinement is the use of a bevelled "teflon" catheter introduced over the needle, for catheterization of the aorta after the needle is withdrawn (Levy et al., 1962). Left ventricular puncture is a 
straightforward technique that can be quickly accomplished and is nearly always successful. Unfortunately there is a significant risk of hæmopericardium, and a mortality of about 2 per cent is reported by Fleming et al. (1958) and Björk and Lodin (1959).

Transseptal catheterization of the left atrium has been used for left ventricular catheterization, a curved "teflon" catheter being passed over the stylet and advanced through the mitral valve. Brockenbrough, Braunwald, and Ross (1962) found it possible to catheterize the left ventricle in 143 of 151 attempts and described left ventricular cardioangiography by this route. Transseptal catheterization is not without risk, however, and although the left ventricular pressure is recorded it is not possible to obtain a withdrawal tracing across the aortic valve or to record a central aortic pressure tracing unless a peripheral artery is catheterized as well.

It is difficult to make an objective comparison of the three techniques available for investigating aortic stenosis. Retrograde arterial catheterization of the ventricle does however compare favourably with percutaneous left ventricular puncture on the grounds of safety, and with transseptal catheterization in that withdrawal pressure records across the aortic valve are obtained. The disadvantages of the technique are that it was only successful in 85 per cent of patients and that it was sometimes rather time consuming. It is probably the method of choice in congenital aortic stenosis when the stenosis is often not at the valve and when gradients at two levels are sometimes found. It is in these patients that it is most helpful to be able to compare the findings at cardioangiography with the pressure tracings.

\section{SUMmaRY}

Twenty-five patients with various types of aortic stenosis were investigated by retrograde arterial catheterization of the left ventricle. Quite a large catheter was used, introduced by arteriotomy. The catheter entered the left ventricle in 21 of the 25 patients, and the pressure tracings obtained indicated clearly the pressure gradient between the aorta and left ventricle and also differentiated between stenosis at different sites.

Cardioangiography after injection of contrast medium into the left ventricle helped in elucidating the nature of the obstruction and also demonstrated the degree of mobility of the aortic valve in valvar stenosis. It was often difficult to carry out the procedure in patients with calcific valvar stenosis, but in other patients the passage of the catheter was readily accomplished. There were no complications in this series and the technique seems to compare favourably with other means of investigating aortic stenosis.

We are indebted to our colleagues for their critical interest in this work and in particular to Dr. J. C. A. Raison and to Dr. J. Mackinnon who have carried out some of the investigations and to Dr. C. Ward, our anæsthetist. We are also grateful to Dr. J. Wearing and to the staff of the Radiological Department for their skilful assistance and to Miss J. Brooks for her technical aid. Miss A. L. Hebden of the Coventry and Warwickshire Hospital kindly prepared the illustrations.

\section{REFERENCES}

Björk, V. O., and Lodin, H. (1959). Left heart catheterization with selective left atrial and ventricular angiocardiography in the diagnosis of mitral and aortic valvular disease. Progr. cardiovasc. Dis., 2, 116.

Brock, R., Milstein, B. B., and Ross, D. N. (1956). Percutaneous left ventricular puncture in the assessment of aortic stenosis. Thorax, 11, 163.

Brockenbrough, E. C., Braunwald, E., and Ross, J. (1962): Transseptal left heart catheterization. A review of 450 studies and description of an improved technic. Circulation, 25, 15.

Fleming, H. A., Hancock, E. W., Milstein, B. B., and Ross, D. N. (1958). Percutaneous left ventricular puncture with catheterization of the aorta. Thorax, 13,97.

Gray, I. R., Joshipura, C. A., and Mackinnon, J. (1963). Retrograde left ventricular cardioangiography in the diagnosis of mitral regurgitation. Brit. Heart J., 25, 145.

Gründemann, A. M., Bosch, G. A. C., Schwantje, E. J. M., Reijns, G. A., and Verheugt, A. P. M. (1960). Retrograde catheterization of the left ventricle in aortic stenosis. Amer. J. Cardiol., 6, 915.

Levy, M. J., Amplatz, K., and Lillehei, C. W. (1962). Transthoracic left heart catheterization and angiocardiography for combined assessment of mitral and aortic valves. Radiology, 78, 638.

Prioton, J.-B., Thévenet, A., Pelissier, M., Puech, P., Latour, H., and Pourquier, J. (1957). Cardiographie ventriculaire gauche par cathétérisme rétrograde percutané fémorale. Presse méd., 65, 1948.

Stampbach, O., and Joss, E. (1959). Der Katheterismus des Linken Herzens. Cardiologia (Basel), 35, 382.

Zimmerman, H. A., Scott, R. W., and Becker, N. O. (1950). Catheterization of the left side of the heart in man. Circulation, 1, 357.

$\mathbf{P}$ 